\title{
How to detour Treg cells in T cell-based antitumor immune therapy
}

\author{
This article was published in the following Dove Press journal: \\ OncoTargets and Therapy \\ 5 September 2013 \\ Number of times this article has been viewed
}

\author{
Shu Zheng' \\ Yanwei Shen ${ }^{1,2}$ \\ Yongmao Song ${ }^{1,3}$ \\ Ying Yuan ${ }^{1,2}$ \\ 'The Cancer Institute, Key \\ Laboratory of Cancer Prevention and \\ Intervention China National Ministry \\ of Education, Key Laboratory of \\ Molecular Biology in Medical Sciences, \\ Zhejiang Province, ${ }^{2}$ Department \\ of Medical Oncology, ${ }^{3}$ Department \\ of Surgical Oncology, The Second \\ Affiliated Hospital, Zhejiang University \\ School of Medicine, Hangzhou, \\ People's Republic of China
}

\begin{abstract}
T cell-based antitumor immune therapy which occupies the boosting area of translational medicine research is capable of eradicating some kinds of tumors that are in late stages. However, the effectiveness of adoptive cell transfer treatment varies among the different clinical trials, while the safety of cells is still uncertain for some patients. All these phenomena provoke us to ask whether the instability of $\mathrm{T}$ cell-based antitumor immune therapy is due to immune modulation function of Treg cells in the tumor microenvironment and the peripheral circulation. Some successful Treg-targeting treatments in clinical trials provide the inspiration for subtle modulation of Treg cells in future cancer immunotherapies. We hypothesized that Treg cells may somehow sense the abundance of peripheral immune effector cells, and maintain the shifted tumor-bearing homeostasis of the immune system. Killer cells infused in adoptive cell transfer therapy may be monitored and spontaneously downregulated by Treg cells. Further studies are required to develop more effective combinations of immunotherapy with conventional chemo/radiotherapy in the modulation of immune-suppressive cells.
\end{abstract}

Keywords: regulatory T lymphocytes, Treg cells, adoptive cell transfer, tumor immune tolerance, immune modulation, cytokine induction

\section{Introduction}

With improvement in our understanding of tumor biology and immunology in recent decades, the importance of inflammation during tumorigenesis and treatment has been emphasized as a hallmark of cancer. The complexity of the tumor microenvironment is formed not only by cancer cells, stromal cells, and endothelial cells, but also by inflammatory cells. Interestingly, these inflammatory cells operate in conflicting ways, with both tumor-antagonizing and tumor-promoting leukocytes found in various proportions. ${ }^{1}$ Conventional therapeutic strategies for cancer have been surgery, radiotherapy, and chemotherapy for centuries, but a fourth modality of immunotherapy has been well documented since 1890 when Coley demonstrated that bacterial products (Coley toxins) had benefits for inoperable breast cancer. ${ }^{2}$ Another milestone in cancer immunotherapy was the finding of an interaction between dying tumor cells caused by conventional anticancer therapy and activation of the innate immune system. Therefore, the efficacy of anticancer treatments should no longer be assessed by their ability to inhibit proliferation of tumor cells directly, but also by their potential to trigger an immunoadjuvant pathway. ${ }^{3}$ Here we review the relevant literature concerning the progress of anticancer immunotherapy, and propose our hypothesis for future strategies involving a combination of conventional therapies and immunotherapy. 


\section{Current limitations of antitumor immune therapy}

Cancer immunotherapy is not only the boosting area of basic scientific research for immunologists, but also a frontier of translational medicine for physicians. Application of immunotherapy is not just a complementary method for traditional treatments, but is a brand new way of clinical thinking. To date, safety and effectiveness are two key problems limiting its clinical application.

Early attempts in anticancer immune therapy include tumor antigen protein vaccination, the GVAX cancer vaccine (BioSante Pharmaceuticals, Baudette, MN, USA) the dendritic cell vaccine, and adoptive $\mathrm{T}$ cell transfer. ${ }^{4}$ There were several Phase I clinical trials of tumor antigen peptide vaccination reported for solid tumors, but the Phase III trials were still needed for further confirmation of the efficacy of tumor antigen peptide vaccination. ${ }^{5-7}$ The GVAX vaccination was started in the early 2000s, and was based on allogenic cancer cell lines transfected with granulocyte-macrophage colony-stimulating factor, which could recruit autologous antigen-presenting cells to the injection site, and maturation of dendritic cells primed host $\mathrm{CD} 4^{+}$and $\mathrm{CD} 8^{+} \mathrm{T}$ cells to generate tumor-specific $\mathrm{T}$ cells for eradication of the host tumor. The combination of two prostate cancer cell lines (PC3 and $\mathrm{LNCaP}$ ), which were engineered via adenovirus to produce granulocyte-macrophage colony-stimulating factor, were tested in clinical trials. While it showed promise in singlearm Phase II studies, the subsequent two Phase III studies did not achieve the expected improvements in survival. ${ }^{8}$ The dendritic cell vaccine is another promising strategy for immunotherapy. This vaccine was generated from autologous peripheral blood mononuclear cells of patients and challenged with specific cancer antigen ex vivo for maturation. After inoculation, the dendritic cell vaccine was capable of activating and inducing replication of antigen-specific immune $T$ cells to recognize and kill antigen-positive cancer cells. The landmark approval of sipuleucel-T (Provenge ${ }^{\circledR}$, Dendreon Corporation, Seattle, WA, USA) on April 29, 2010 was an important breakthrough for the affirmative vote from the US Food and Drug Administration to cell therapy other than conventional chemical agents. This therapeutic cancer vaccine demonstrated prolonged overall survival (25.8 months versus 21.7 months in the placebo arm; $P=0.017$ ) in Phase III trials of advanced hormonerefractory prostate cancer. ${ }^{9}$

There are several strategies for $\mathrm{T}$ cell-based immune therapy, namely lymphokine-activated killer cell, tumor-infiltrating lymphocyte (TIL), CD8 ${ }^{+}$cytotoxic $\mathrm{T}$ lymphocyte, and cytokine-induced killer cell (CIK) adoptive cell transfer. As early as the early 1980 s, several research groups had already started using adoptive cell transfer of lymphokine-activated killer cells to treat cancer patients. ${ }^{10}$ The most popular cancer immunotherapy today is TIL adoptive transfer, developed in the late 1980s. ${ }^{11}$ TIL adoptive transfer has shown much promise in the treatment of melanoma and renal cell carcinoma. It has been reported that renal cell carcinoma can be effectively controlled by immunologic manipulation using adoptive cell transfer treatment. ${ }^{12}$ Melanoma is another sample of efficient immunotherapy eradicating a disseminated cancer. ${ }^{13,14}$

\section{Reasonable explanation for failure of adoptive cell transfer}

Cancerous disease is characterized by successful escape from host immune surveillance, ${ }^{1}$ which would be achieved by recruiting immune suppressive cells in the tumor microenvironment . Immune suppressive cells discovered in the tumor microenvironment included tolerogenic dendritic cells, tumor-associated macrophages, and regulatory $\mathrm{T}$ lymphocytes (Treg cells). In cancer patients, Treg cell percentages were found to be elevated and this increase correlated with disease progression and worsened prognosis. ${ }^{15}$ Other myeloid-derived suppressive cells newly discovered in recent years have already become a hot topic in translational medicine research. ${ }^{16}$ The existence of these immune suppressive cells in the circulation and in tumor-infiltrating cells challenges the safety and effectiveness of existing immunotherapy. It is critical to improve the purity of killer cells, meanwhile overcoming disturbance from suppressive cells.

Although TIL adoptive transfer therapy has a therapeutic effect in melanoma and renal cell carcinoma, the heterogeneity of TIL cells is a major obstacle to its clinical application. TIL cells consist of different $\mathrm{T}$ cell populations, including suppressive regulatory $\mathrm{T}$ cells, which account for $5 \%-10 \%$ of $\mathrm{CD}^{+}{ }^{+} \mathrm{T}$ lymphocytes under normal conditions. ${ }^{17}$ It is widely accepted that some cases of immunotherapy failure may be due to accumulation of Treg cells in the peritumoral regional of the tumor microenvironment as well as in the peripheral circulation. ${ }^{18}$ One research group reported that, during autologous nodal $\mathrm{T}$ cell adoptive transfer from patients' normal lymph nodes and allogeneic $\mathrm{CD} 8^{+} \mathrm{CD} 25^{-}$ and $\mathrm{CD} 4^{+} \mathrm{CD} 25^{-} \mathrm{T}$ cells from normal donor peripheral blood mononuclear cells, $\mathrm{CD} 4^{+} \mathrm{CD} 25^{+} \mathrm{GITR}^{+}$Treg cells in the lymph nodes of patients were capable of suppressing proliferation of $T$ effector cells via the transforming growth factor- $\beta$ signaling pathway. ${ }^{19}$ Another research team reported that Treg cells could also impair natural killer cell-based treatment of lung 
cancer, which could be partially rescued by blockade of GITR and transforming growth factor- $\beta 1 .^{20}$ Thus, the existence of Treg cells and other suppressive immune cells in the tumor microenvironment is a major obstacle for current antitumor immunotherapy strategies.

On the other hand, it has been known since the 1990s that conventional chemotherapy can augment the host immune reaction against tumor tissue due to release of tumor antigen from dead cells. ${ }^{21} \mathrm{~A}$ recent paper demonstrated that chemotherapeutic agents not only have a cytotoxic effect but also harness the host immune system, contributing to their antitumor activity. ${ }^{22}$ Chemotherapy combined with immunotherapy has been reported to enhance the antitumor response and to have therapeutic advantages over single modality treatment. ${ }^{23}$ Interestingly, cyclophosphamide is a chemotherapeutic agent with a dose-dependent, bimodal effect on the immune system. One injection of low-dose cyclophosphamide $(100 \mathrm{mg} / \mathrm{kg}$ of body weight in mouse, comparing to the chemotherapeutic dose of 200-300 mg/kg in mice), administered intraperitoneally and given three days prior to vaccination, was able to decrease Treg cell numbers and also inhibit their suppressive capability. ${ }^{24}$ Therefore, cyclophosphamide could enhance antitumor therapeutic efficiency due to its immune-modulating effect in confining Treg cell suppression.

\section{Tumor defense barrier hypothesis}

According to this hypothesis, immune cells recruited in the tumor microenviroment block the attack on exogenous delivery of killer cells, and this is supported by current mouse level data and clinical findings. Further, Treg cells may somehow sense abundance of peripheral immune effector cells and keep the tumor-bearing shifted homeostasis of the immune system inside the body. Killer cells delivered into the patient may be considered to be excessive by Treg cells, which would be spontaneously enhanced to shut down the pseudoautoimmune reaction.

\section{Evidence of the barrier hypothesis}

During allogeneic bone marrow/stem cell transplant procedures for acute myelogenous leukemia, the effect of bone marrow/stem cell transplant has been found to be better during the chemotherapy releasing stage, and there has been a report that aged neutrophils home back into the bone marrow and promote reduction in the size and function of the hematopoietic niche. ${ }^{25}$ This phenomena prompted us to consider Treg cells as the monitoring cells which travel back to the central immune organs in order to control the production of $\mathrm{T}$ effector cells. This is most likely to be regulatory
$\mathrm{T}$ cells that function in maintaining the effector $\mathrm{T}$ cells so they do not exceed the capacity of host immune organs.

As mentioned above, it has been known for about 20 years that the chemotherapeutic agent cyclophosphamide is able to abolish Treg cells and thereby enhance the antitumor immune response. Several clinical trials of the use of immunotherapy in combination with cyclophosphamide in different kinds of solid tumors have been reported. ${ }^{25-29}$ Other strategies for blocking Treg cells have already been used in clinical trials of antitumor immunotherapy, ${ }^{29-31}$ but surprisingly, the fusion protein of interleukin-2 and diphtheria toxin (DAB389IL-2, Ontak $^{\mathrm{TM}}$, Eisai Inc, Woodcliff Lake, NJ, USA) targeting Treg cells had disappointing results in eliminating Treg cells in patients with metastatic melanoma. ${ }^{28}$ Further, daclizumab (an anti-CD25 monoclonal antibody) and ipilimumab (an anticytotoxic T lymphocyte A4 antibody) have shown some effect on restraining Treg cell suppressive function, resulting in an enhanced immune response to tumor antigen vaccination, but with a large number of side effects as well. ${ }^{32}$ Clinical investigation of PD-1, another immune checkpoint cell surface marker, has also shown potential in clinical application. ${ }^{33}$ However, excessive inhibition of Treg cells may cause an autoimmune response in terms of allergic reactions and pulmonary hyper-reactivity, and the Treg cells would bounce back even more after medication with the drugs listed above. Therefore, rather than radical elimination of Treg from the body, we proposed shifting the Treg percentage or the functional effectivity of Treg downwards in order to break through the "barrier" of the tumor.

\section{How to elicit the hypothesis and detour the Treg barrier}

Because of the existence of immune suppressive cells in the peripheral circulation and the tumor microenvironment, the effect of antitumor immune therapy is diminished, which limits its application in the clinic. We hypothesized that increased Treg cells in the peripheral circulation of cancer patients from the local tumor environment is designated for sensing immune homeostasis inside the body. If we directly deliver abundant $\mathrm{T}$ effector cells into the patient, the Treg cells would take them as an excessive autoimmune reaction and spontaneously enhance themselves to diminish those intruders. Because of the great side effects of complete elimination of Treg cells through monoclonal antibodies, the side-effects of autoimmune response are an obstacle for clinical application of these therapeutic drugs. Further, if chemotherapy is stopped, the Treg cells would still bounce back to the former level due to the natural Treg differentiated 
from lymphoid progenitor cells. However, if we could break through the Treg barrier in the tumor microenvironment, we would surely achieve greater advances in antitumor immune therapy. There are already some published papers indicating that antitumor adoptive cell transfer therapy combined with eradication of Treg cells could achieve a better therapeutic effect. Zhou et al reported that depletion of endogenous Treg cells could improve the efficacy of adoptively transferred tumor-reactive cytotoxic T lymphocytes in a murine model of acute myeloid leukemia. ${ }^{34}$ Natural killer cell-based adoptive transfer therapy could also be enhanced by Treg depletion, ${ }^{35}$ as in the murine solid tumor treatment model.

\section{Discussion}

$\mathrm{T}$ cell-based antitumor immune therapy has shown great promise in melanoma and renal cell carcinoma, and how to broaden its application to the treatment of other solid tumors is the next question. The safety and effectiveness of cancer immunotherapy is always the first consideration before we apply the fundamental research to translational medicine.

One of the well established hallmarks of cancer is its ability to evade immune destruction, ${ }^{1}$ which is accomplished by recruitment of suppressive immune cells in the tumor microenviroment. These immune suppressive cells would sequentially block attack from delivered killer cells, but their success in leukemia bone marrow transplantation provokes us to dissect into the balancing between tumor-suppressive effect and total immune cell overload. Is the Treg cell able to sense the peripheral abundance of effector immune cells, and would the load of effector cells have feedback on Treg function? Does the increased Treg portion in the peripheral blood of patients mean shifted homeostasis of the immune system due to the tumor-bearing status of the body? Radical elimination of Treg cells in patients may have some therapeutic effect, but may also be accompanied by an allergic autoimmune side effect problem and bouncing back of Treg levels after the medication.

All the phenomena listed above led us to generate our hypothesis that Treg cells somehow monitor the load of peripheral immune effector cells, and maintain the shifted tumor-bearing homeostasis of the immune system inside the body. Killer cells delivered into the patient may be considered as excessive overload to the immune system by Treg cells, which would be spontaneously enhanced to shut down the pseudoautoimmune reaction.

If proven to be true, the insights of our hypothesis for clinical oncologists may fall into criteria as follows. First, monitoring the percentage of Treg cells or other immune suppressive cells in peripheral blood may provide the clue for prediction of tumor volume and therapeutic response. Second, chemotherapy or radiotherapy should be applied prior to anticancer immunotherapy in order to decrease the total number of immune cells and make space for the transferred cells. This would also prevent the bone marrow from generating Treg cells in response to delivery of the killer cells. Third, destruction of the Treg barrier in the tumor environment is necessary to allow entry of killer cells from the circulation into the tumor site. In the meantime, the number of cells infused needs to be subtle enough to be sustained under the alarming level of Treg cells. Otherwise, the overloaded effector cells may cause autoimmune reactions and other side effects. Last but not least, use of immunotherapy should cooperate well with conventional chemotherapy and radiotherapy, which are proven to be capable of augmenting the effect of immunotherapy in order to maximize the benefit for patients.

\section{Acknowledgments}

This work was supported by grants from the National Natural Science Foundation of China (81102013), International Science and Technology Cooperation Key Program of Zhejiang Province (2009C14010), and the Zhejiang Provincial Education Ministry (N20110100).

\section{Disclosure}

The authors report no competing financial interests in this work.

\section{References}

1. Hanahan D, Weinberg RA. Hallmarks of cancer: the next generation. Cell. 2011;144(5):646-674.

2. Nauts HC, Fowler GA, Bogatko FH. A review of the influence of bacterial infection and of bacterial products (Coley's toxins) on malignant tumors in man; a critical analysis of 30 inoperable cases treated by Coley's mixed toxins, in which diagnosis was confirmed by microscopic examination selected for special study. Acta Med Scand Suppl. 1953;276:1-103.

3. Apetoh L, Tesniere A, Ghiringhelli F, Kroemer G, Zitvogel L. Molecular interactions between dying tumor cells and the innate immune system determine the efficacy of conventional anticancer therapies. Cancer Res. 2008;68(11):4026-4030.

4. Kirkwood JM, Butterfield LH, Tarhini AA, Zarour H, Kalinski P, Ferrone S. Immunotherapy of cancer in 2012. CA Cancer J Clin. 2012; 62(5):309-335.

5. Hueman MT, Dehqanzada ZA, Novak TE, et al. Phase I clinical trial of a HER-2/neu peptide (E75) vaccine for the prevention of prostate-specific antigen recurrence in high-risk prostate cancer patients. Clin Cancer Res. 2005;11(20):7470-7479.

6. Sabbatini P, Tsuji T, Ferran L, et al. Phase I trial of overlapping long peptides from a tumor self-antigen and poly-ICLC shows rapid induction of integrated immune response in ovarian cancer patients. Clin Cancer Res. 2012;18(23):6497-6508. 
7. Dai S, Wei D, Wu Z, et al. Phase I clinical trial of autologous ascitesderived exosomes combined with GM-CSF for colorectal cancer. $\mathrm{Mol}$ Ther. 2008;16(4):782-790.

8. Simons JW, Sacks N. Granulocyte-macrophage colony-stimulating factor-transduced allogeneic cancer cellular immunotherapy: the GVAX vaccine for prostate cancer. Urol Oncol. 2006;24(5):419-224.

9. Kantoff PW, Higano CS, Shore ND, et al. Sipuleucel-T immunotherapy for castration-resistant prostate cancer. $N$ Engl J Med. 2010;363(5):411-422.

10. Eberlein TJ, Rosenstein M, Rosenberg SA. Regression of a disseminated syngeneic solid tumor by systemic transfer of lymphoid cells expanded in interleukin 2. J Exp Med. 1982;156(2):385-397.

11. Rosenberg SA, Packard BS, Aebersold PM, et al. Use of tumorinfiltrating lymphocytes and interleukin-2 in the immunotherapy of patients with metastatic melanoma. A preliminary report. $N$ Engl J Med. 1988;319(25):1676-1680.

12. Gouttefangeas C, Stenzl A, Stevanovic S, Rammensee HG. Immunotherapy of renal cell carcinoma. Cancer Immunol Immunother. 2007;56(1):117-128.

13. Rosenberg SA, Dudley ME. Adoptive cell therapy for the treatment of patients with metastatic melanoma. Curr Opin Immunol. 2009;21(2): 233-240.

14. Mackensen A, Meidenbauer N, Vogl S, Laumer M, Berger J, Andreesen R. Phase I study of adoptive T-cell therapy using antigenspecific CD8+ T cells for the treatment of patients with metastatic melanoma. J Clin Oncol. 2006;24(31):5060-5069.

15. Petersen RP, Campa MJ, Sperlazza J, et al. Tumor infiltrating Foxp3+ regulatory T-cells are associated with recurrence in pathologic stage I NSCLC patients. Cancer. 2006;107(12):2866-2872.

16. Sun HL, Zhou X, Xue YF, et al. Increased frequency and clinical significance of myeloid-derived suppressor cells in human colorectal carcinoma. World J Gastroenterol. 2012;18(25):3303-3309.

17. Valmori D, Merlo A, Souleimanian NE, Hesdorffer CS, Ayyoub M. A peripheral circulating compartment of natural naive CD4 Tregs. J Clin Invest. 2005;115(7):1953-1962.

18. Liu Z, Kim JH, Falo LD Jr, You Z. Tumor regulatory T cells potently abrogate antitumor immunity. J Immunol. 2009;182(10):6160-6167.

19. Hilchey SP, De A, Rimsza LM, Bankert RB, Bernstein SH. Follicular lymphoma intratumoral CD4+CD25+GITR+ regulatory $\mathrm{T}$ cells potently suppress CD3/CD28-costimulated autologous and allogeneic CD8+CD25- and CD4+CD25- T cells. J Immunol. 2007;178(7): 4051-4061.

20. Li H, Yu JP, Cao S, et al. CD4 +CD25 + regulatory T cells decreased the antitumor activity of cytokine-induced killer (CIK) cells of lung cancer patients. J Clin Immunol. 2007;27(3):317-326.

21. Weisenthal LM, Dill PL, Pearson FC. Effect of prior cancer chemotherapy on human tumor-specific cytotoxicity in vitro in response to immunopotentiating biologic response modifiers. J Natl Cancer Inst. 1991;83(1):37-42.
22. Bruchard M, Mignot G, Derangère V, et al. Chemotherapy-triggered cathepsin B release in myeloid-derived suppressor cells activates the Nlrp3 inflammasome and promotes tumor growth. Nat Med. 2013;19(1): 57-64.

23. Ko HJ, Kim YJ, Kim YS, et al. A combination of chemoimmunotherapies can efficiently break self-tolerance and induce antitumor immunity in a tolerogenic murine tumor model. Cancer Res. 2007;67(15): 7477-7486.

24. Lutsiak ME, Semnani RT, De Pascalis R, Kashmiri SV, Schlom J, Sabzevari $\mathrm{H}$. Inhibition of CD4(+)25+ T regulatory cell function implicated in enhanced immune response by low-dose cyclophosphamide. Blood. 2005;105(7):2862-2868.

25. Casanova-Acebes M, Pitaval C, Weiss LA, et al. Rhythmic modulation of the hematopoietic niche through neutrophil clearance. Cell. 2013;153(5):1025-1035.

26. Alfaro C, Perez-Gracia JL, Suarez N, et al. Pilot clinical trial of type 1 dendritic cells loaded with autologous tumor lysates combined with GM-CSF, pegylated IFN, and cyclophosphamide for metastatic cancer patients. J Immunol. 2011;187(11):6130-6142.

27. Walter S, Weinschenk T, Stenzl A. Multipeptide immune response to cancer vaccine IMA901 after single-dose cyclophosphamide associates with longer patient survival. Nat Med. 2012;18(8). Epub 2012 Jul 29.

28. Foss FM. DAB(389)IL-2 (Ontak): a novel fusion toxin therapy for lymphoma. Clin Lymphoma. 2000;1(2):110-116.

29. Walter S, Weinschenk T, Reinhardt C, Singh-Jasuja H. Single-dose cyclophosphamide synergizes with immune responses to the renal cell cancer vaccine IMA901. Oncoimmunology. 2013;2(1):e22246.

30. Olsen E, Duvic M, Franken A, et al. Pivotal phase III trial of two dose levels of denileukin diftitox for the treatment of cutaneous T-cell lymphoma. J Clin Oncol. 2001;19(2):376-388.

31. Duvic M, Kuzel TM, Olsen EA, et al. Quality-of-life improvements in cutaneous T-cell lymphoma patients treated with denileukin diftitox (ONTAK). Clin Lymphoma. 2002;2(4):222-228.

32. Yang JC, Hughes M, Kammula U, et al. Ipilimumab (anti-CTLA4 antibody) causes regression of metastatic renal cell cancer associated with enteritis and hypophysitis. J Immunother. 2007;30(8):825-830

33. Wolchok JD, Kluger H, Callahan MK, et al. Nivolumab plus ipilimumab in advanced melanoma. $N$ Engl J Med. 2013;369(2):122-133.

34. Zhou Q, Bucher C, Munger ME, et al. Depletion of endogenous tumor-associated regulatory $\mathrm{T}$ cells improves the efficacy of adoptive cytotoxic T-cell immunotherapy in murine acute myeloid leukemia. Blood. 2009;114(18):3793-3802.

35. Lundqvist A, Yokoyama H, Smith A, Berg M, Childs R. Bortezomib treatment and regulatory T-cell depletion enhance the antitumor effects of adoptively infused NK cells. Blood. 2009;113(24):6120-6127.
OncoTargets and Therapy

\section{Publish your work in this journal}

OncoTargets and Therapy is an international, peer-reviewed, open access journal focusing on the pathological basis of all cancers, potential targets for therapy and treatment protocols employed to improve the management of cancer patients. The journal also focuses on the impact of management programs and new therapeutic agents and protocols on

\section{Dovepress}

patient perspectives such as quality of life, adherence and satisfaction The manuscript management system is completely online and includes a very quick and fair peer-review system, which is all easy to use. Visit http://www.dovepress.com/testimonials.php to read real quotes from published authors. 